\title{
On-Line Non-Contact System for Grinding Wheel Wear Measurement
}

\author{
K.-C. Fan ${ }^{1}$, M.-Z. Lee ${ }^{1}$ and J.-I. Mou ${ }^{2}$ \\ ${ }^{1}$ Department of Mechanical Engineering, National Taiwan University, Taipei, Taiwan; and ${ }^{2}$ Department of Industrial Engineering, \\ Arizona State University, Tempe, Arizona, USA
}

\begin{abstract}
An on-line non-contact method for measuring the wear of a form grinding wheel is presented. A CCD (charge coupled device) camera with a selected optical lens and a frame grabber was used to capture the image of a grinding wheel. The analogue signals of the image were transformed into corresponding digital grey level values. Using the binarisation technique, the images of background and the grinding wheel were segmented. Thus the grinding wheel edge was identified. The 'mapping function method' is used to transform an image pixel coordinate to a space coordinate. An auto-focus technology is also developed. The statistics of pixels are used as the focusing index. The signal was sent through an 8255 control card to drive a d.c. motor, and then to control the lens focusing movement to acquire the focal plane. The images before and after the grinding process were captured. The position deviation of the grinding wheel edge was analysed. Then, the grinding wheel wear was evaluated. The wear detection accuracy is about $1 \mu \mathrm{m}$.
\end{abstract}

Keywords: Autofocus; Binarisation; CCD; Grinding wheel; Mapping function method; Wear

\section{Introduction}

Since grinding is commonly used as a high-precision process to ensure product quality, the grinding wheel plays an important role in precision machining [1]. Wear will occur on a grinding wheel after lengthy use, as with many other tools. The wear will dull the abrasive particles and reduce the grinding wheel radius, thus, directly affecting the grinding efficiency and dimensional accuracy of the finished parts. Therefore, grinding wheels must be inspected periodically for wear. When wear is severe, dressing and trueing must be applied to ensure the proper sharpness and size.

Correspondence and offprint requests to: Professor K.-C. Fan, Department of Mechanical Engineering, National Taiwan University, No. 1 Roosevelt Road, Sec. 4, Taipei, Taiwan. E-mail: fan@ccms.ntu.edu.tw
The most popular methods for grinding wheel wear monitoring and inspection are acoustic emission testing [2], a power and acoustic emission combination [3], a touch-trigger probe and acoustic emission combination [4], a touch-trigger probe inspection method [5], and an optical triangulation method [6]. The measured signals were converted to an actual wear amount through empirical model interpolation. All these methods are either indirect or off-line measurement.

Our goal is to develop an automatic on-line grinding wheel wear monitoring and inspection system. The developed system uses a CCD (charge coupled device) camera to monitor the image of the wheel edge. By observing the wheel edge images before grinding and after grinding and comparing them with a calibrated wear scale, the actual grinding wheel wear could be determined. Thus, the developed system is a direct and online wear measurement approach.

Tsai [7] used a parametric method to calibrate the CCD camera image distortion in an application for 3D machine vision metrology. Wang [8] proposed a method to calibrate the focus distortion of video camera lenses for an optical measurement system. Ohlander et al. [9] derived a method, according to the characteristic of the analysed image histogram, to set different thresholds that divide the picture or image into several subregions.

To increase the speed of edge detection and the stability of measurement, the system developed in this study uses a binarisation technique with a properly selected threshold to identify quickly the grinding wheel edge. To reduce potential humanrelated error, an autofocus technique is also developed that uses a computer-controlled motor to fine-tune the lens focusing motion and uses a software algorithm to analyse the statistics of the pixels as an index for spot focus.

\section{Image Processing Principles}

\subsection{Digital Image Handling System}

A CCD camera can capture the image of an object visually and present it as a series of analogue RS-170 signals, as shown in Fig. 1. The analogue signal can be converted and stored as 


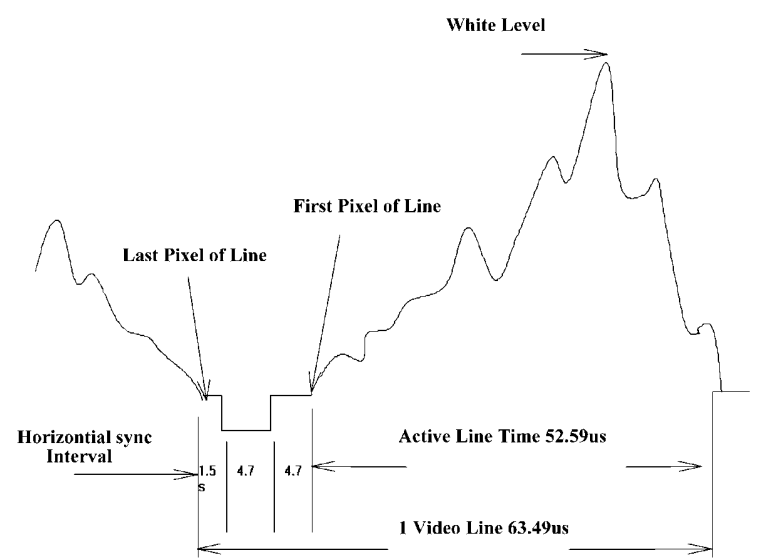

Fig. 1. RS170/330 signal characteristics.

a digital signal using a signal-processing card. Once the image is digitised, the image is replaced by a large set of pixels. Every pixel has a specific coordinate corresponding to a point on the object surface, as shown in Fig. 2. The light intensity of a pixel is defined as a grey level, in the range 0-255 for an 8-bit A/D conversion, of that point on the object surface. The larger the grey level, the higher the light intensity.

For a digitised image, the quality and resolution has a close relationship. There are two types of resolution: space resolution and brightness resolution. The higher the space resolution, the more pixels an image can be divided into. The finer the division, the better the quality of the image. Brightness resolution depicts the pixel's light intensity. The higher the brightness resolution, the easier it is to show the contrast between dark and bright image pixels. As both resolutions increase, the image quality becomes better. However, the required memory space, processing time, and hardware cost also increases.

\subsection{Binarisation Image Processing}

Binarisation is a commonly used technique to handle various grey level pixels. With a predefined grey level threshold, the

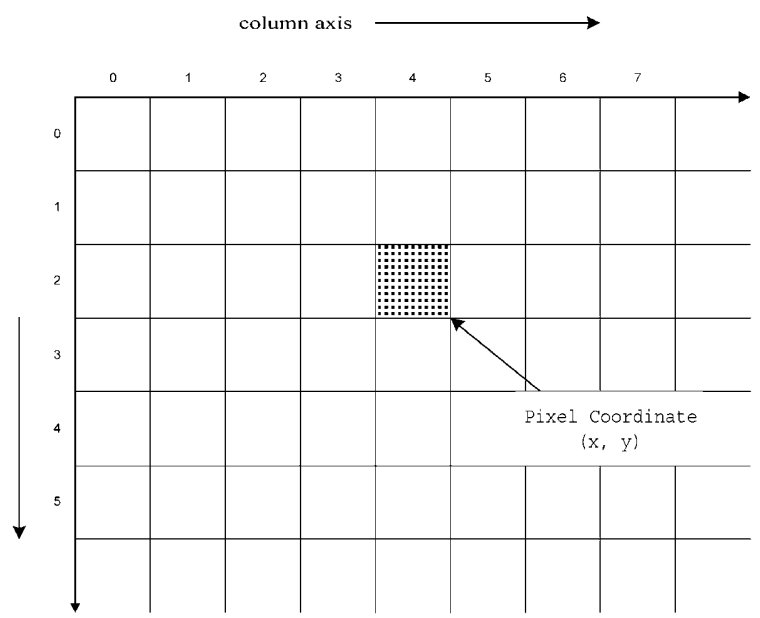

Fig. 2. A digitised image pixel and its corresponding monitor coordinates.

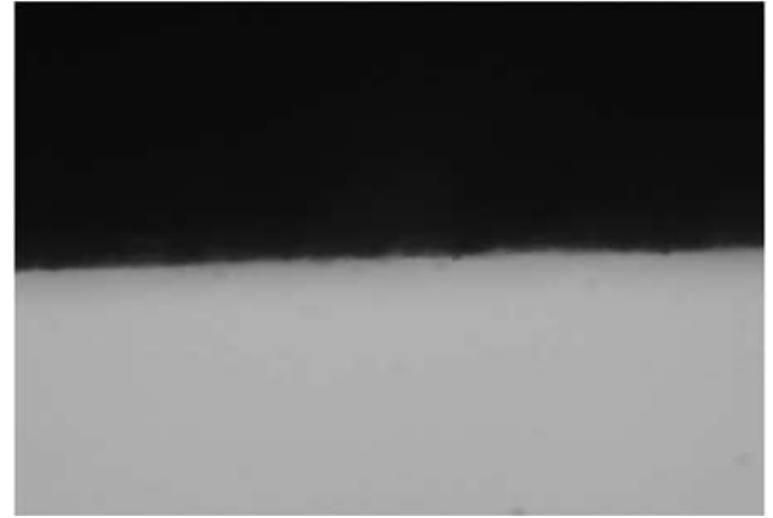

Fig. 3. Grinding wheel edge image.

technique can categorise pixels as either totally black or totally white. Mathematically, binarisation can be expressed as follows [10].

$$
P\left(g_{q}\right)=\left\{\begin{array}{cl}
0 & \left(g_{q}<t\right) \\
255 & \left(g_{q} \geq t\right)
\end{array}\right.
$$

where $t$ is the threshold, $g_{q}$ is the grey level of point $q$, and $P$ is a point operation.

As an example, Fig. 3 shows a grinding wheel edge captured by an image processor. The dark section is the grinding wheel and the white section is the background.

The developed system adapts the binarisation technique to detect quickly the edge of grinding wheel. For an image 640 pixels long and 480 pixels wide, the required resolution is 640 $\times 480=307200$ pixels. If binarisation is applied for each pixel, the burden of computation and logic reasoning will be heavy. To avoid this time-consuming computation, a look-up table was adapted. The look-up table is user definable for any specific transformation function. For constant input and output, the table provides a linear transformation, as shown in Fig. 4.

For the developed system, the binarisation was achieved using the following definition.

$$
\begin{aligned}
& g(x)=f(x)-\left[\frac{f(x)}{255}\right] \times 255 \\
& f(x)=a+(x / 10) \quad(x=0 \sim 255)
\end{aligned}
$$

where $x$ is the original grey level, $g(x)$ is the transformed gravel level, [] is a Gaussian function, [f(x)/255] is defined as the largest integer less than $f(x) / 255, a$ is the control parameter

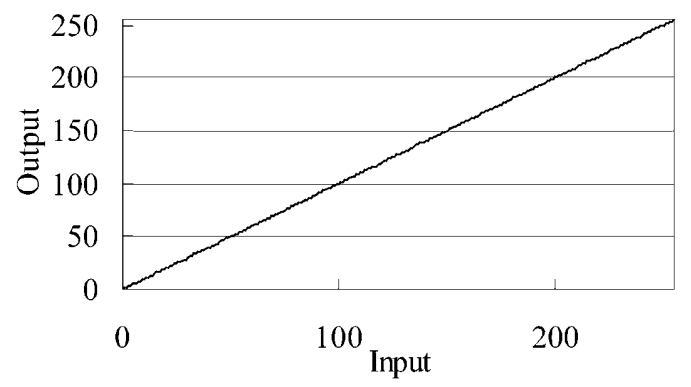

Fig. 4. Linear look-up for transformation table. 


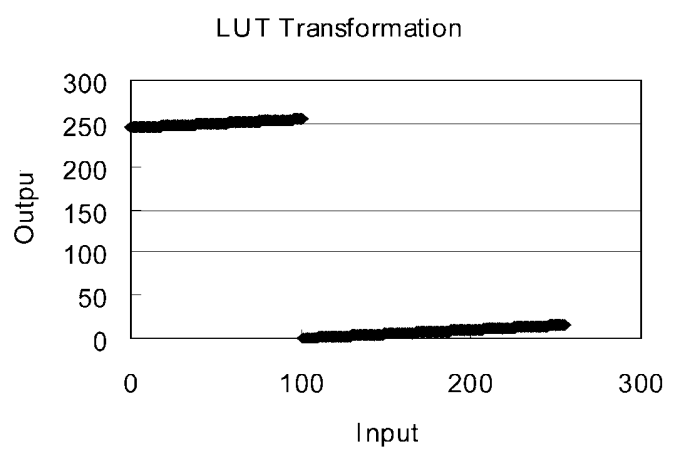

Fig. 5. Look-up table for binarisation transformation.

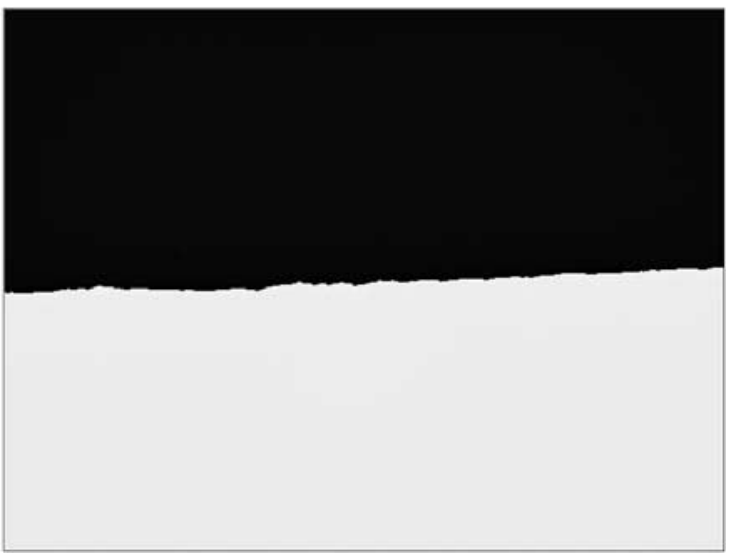

Fig. 6. Grinding wheel edge image after binarisation.

for the threshold. If $a=245$, then the threshold $=100$. The binarisation transformation table is shown in Fig. 5. According to Fig. 5, if the input grey level value is less than 100, the output grey level value will close to 255 (all white). When the input grey level value is larger than 100 , the output grey level value will be close to 0 (all black). Although the transformation will invert the image colour, it does not affect the result of edge finding.

After the binarisation process, a grinding wheel edge image can be shown as a sharp contrast black and white image, as shown in Fig. 6. Figure 7 shows that, along a particular vertical

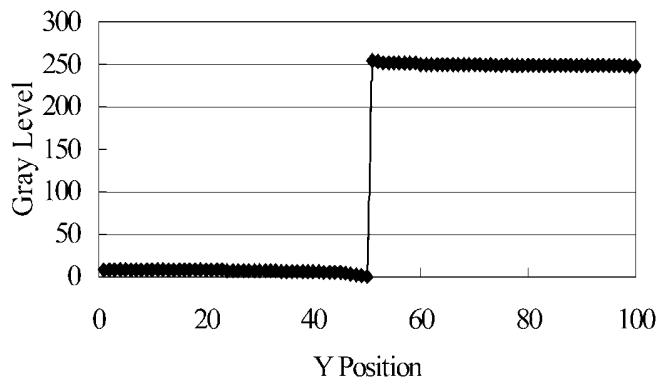

Fig. 7. The grey level and the corresponding $Y$-position after binarisation. line ( $Y$-direction) of an image plane, the location where a grey level varies sharply is the edge location. Therefore, the edge finding process requires only one image transformation and one logic reasoning. Thus, the computational cost can be reduced significantly.

\subsection{Regressions for Line and Circle}

The detected edge image will be compared with the original data to determine the wear value. The least-squares method with the distance from each point to fitted line [11] was used to avoid the potential dilemma of fitting a vertical line.

The principle is to find the coordinate of the data mean and the angle between a straight line passing through the mean coordinate and the $x$-axis. The equation for that straight line is expressed as follows:

$$
\left(y_{i}-y_{0}\right) \cos \theta-\left(x_{i}-x_{m}\right) \sin \theta=0
$$

where $y_{0}$ and $\theta$ are the parameters, $x_{m}$ is the $x$-axis mean coordinate.

Let $S$ be the sum of the square of errors and be expressed as follows:

$$
S=\sum_{i=1}^{n} d_{i}^{2}=\sum_{i=1}^{n}\left(\left(y_{i}-y_{0}\right) \cos \theta-\left(x_{i}-x_{m}\right) \sin \theta\right)^{2}
$$

Taking the partial differentiation of $S$ with respect to $y_{0}$ and $\theta$, and letting $\partial s / \partial y_{0}=0$ and $\partial S / \partial \theta=0$, yields

$$
\begin{aligned}
y_{0} & =\frac{1}{n} \sum_{i=1}^{n} y_{i}=y_{m}, \\
\theta & =\frac{1}{2} \tan ^{-1}\left[\frac{2 I_{x y}}{I_{x}-I_{y}}\right]
\end{aligned}
$$

where,

$$
\begin{aligned}
I_{x y} & =\sum_{i=1}^{n}\left(x_{i}-x_{m}\right)\left(y_{i}-y_{m}\right) \\
I_{x} & =\sum_{i=1}^{n}\left(x_{i}-x_{m}\right)^{2} \\
I_{y} & =\sum_{i=1}^{n}\left(y_{i}-y_{m}\right)^{2}
\end{aligned}
$$

Thus, $y_{0}=y_{m}$ is the $y$-axis mean coordinate and $\theta$ is the angle between the straight line passing through the mean and the $x$-axis. When $I_{x}=I_{y}, \theta=45^{\circ}$.

The equation for the best-fit circle in which the sum of squares of the radial distance of each point on the circle is the smallest can be expressed as follows:

$$
x^{2}+y^{2}+A x+B y+C=0
$$

The objective function, $S$, becomes

$$
S=\sum_{i=1}^{n} \delta_{i}^{2}=\sum_{i=1}^{n}\left(x_{i}^{2}+y_{i}^{2}+A x_{i}+B y_{i}+C\right)^{2}
$$


Taking the partial differentiation of $S$ with respect to $A, B$, and $C$, yields the following equations:

$$
\begin{aligned}
& \frac{\partial S}{\partial A}=\sum_{i=1}^{n} 2\left(x_{i}^{2}+y_{i}^{2}+A x_{i}+B y_{i}+C\right)\left(x_{i}\right)=0 \\
& \frac{\partial S}{\partial B}=\sum_{i=1}^{n} 2\left(x_{i}^{2}+y_{i}^{2}+A x_{i}+B y_{i}+C\right)\left(y_{i}\right)=0 \\
& \frac{\partial S}{\partial C}=\sum_{i=1}^{n} 2\left(x_{i}^{2}+y_{i}^{2}+A x_{i}+B y_{i}+C\right)(1)=0
\end{aligned}
$$

Rearranging those equations, yields

$$
\left[\begin{array}{ccc}
\Sigma x_{i}^{2} & \Sigma x_{i} y_{i} & \Sigma x_{i} \\
\Sigma x_{i} y_{i} & \Sigma y_{i}^{2} & \Sigma y_{i} \\
\Sigma x_{i} & \Sigma y_{i} & 1
\end{array}\right]\left[\begin{array}{l}
A \\
B \\
C
\end{array}\right]=\left[\begin{array}{c}
-\Sigma x_{i}\left(x_{i}^{2}+y_{i}^{2}\right) \\
-\Sigma y_{i}\left(x_{i}^{2}+y_{i}^{2}\right) \\
-\Sigma\left(x_{i}^{2}+y_{i}^{2}\right)
\end{array}\right]
$$

Solve for $A, B$, and $C$, then the minimum sum of the squares for a circle could be derived with the following parameters:

$$
\begin{aligned}
& \left(x_{0}, y_{0}\right)=\left(-\frac{A}{2},-\frac{B}{2}\right) \\
& \text { and } R=\frac{\sqrt{\left(A^{2}+B^{2}-4 C\right)}}{2}
\end{aligned}
$$

\section{CCD Camera Calibration}

Without proper calibration, the relationship between the camera coordinate system and the actual spatial coordinate system would not be known and thus the image dimension represented by a single pixel could be determined. Also, because of lens distortion, the image would be distorted and lead to a measurement error. Therefore, the CCD camera parameters must be calibrated before use.

In general, there are two factors that will cause errors in edge finding. The first is related to CCD camera calibration. The second is related to noise from the surroundings. There are two sets of parameters which must be calibrated for a CCD camera. The extrinsic parameters involve the transformation between the scene coordinates, camera coordinates, image coordinates, and pixel coordinates. The intrinsic parameters involve following internal geometric variables:

1. Camera constant - the distance between the projection centre and the image plane.

2. Principle point - the intersection of optical axis and image plane.

3. Lens distortion - the lens related image distortion.

4. Aspect ratio - the length-width ratio of each pixel.

There are two types of lens distortion: radial distortion and decentring. Radial distortion will cause the incoming light to bend more or less than normal, as shown in Fig. 8. Decentring is the offset between the lens centre and the optical axis. The actual distortion patterns, barrel distortion and pincushion distortion, are shown in Fig. 9.

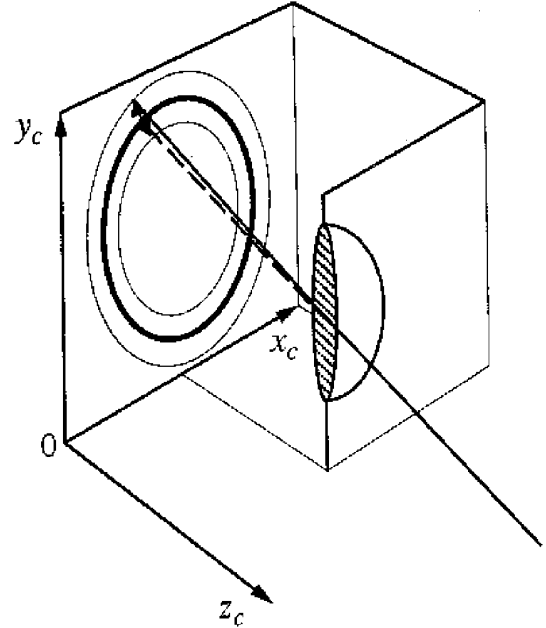

Fig. 8. The radial distortion scheme. (a)

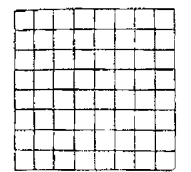

(b)

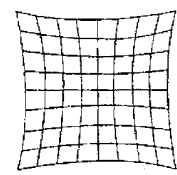

(c)

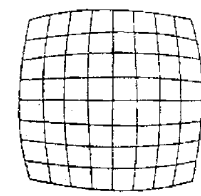

Fig. 9. Common distortion patterns. (a) Original. (b) Pincushion distortion. (c) Barrel distortion.

Tsai [7] derived a method for CCD camera calibration of extrinsic and intrinsic parameters. The distortion coefficients were derived and used to determine the actual image position in the pixel coordinate system. The method can calibrate the CCD camera accurately but is very time consuming and is not suitable for on-line inspection and compensation in a production environment.

To increase the precision of grinding wheel wear monitoring, the lens magnification ratio must be increased significantly. Consequently, the view window will be reduced and the size of the standard mask for calibration must also be small. Coordinate transformation was involved in the derivation of the calibrated distortion coefficient. Owing to the relatively small size mask compared with the distance from the lens to the mask, it is very difficult to detect any change to the coordinates of the mask. Thus, the accuracy of the derived calibrated distortion coefficient may be questionable. An independent measuring instrument such as a laser interferometer is required to provide the calibration transformation scale, but this is very inconvenient for on-line operation.

In this study, the mapping function method proposed by Dai [12] was adapted to derive the relationship between the measuring plane spatial coordinate and CCD camera image coordinate. In this approach, both extrinsic and intrinsic parameter variations are treated as the system error and the distortion coefficients are not solved explicitly.

According to image processing principles, any point in the CCD camera viewing space can be projected to a pixel on the image plane, as shown in Fig. 10. The mapping relationship 


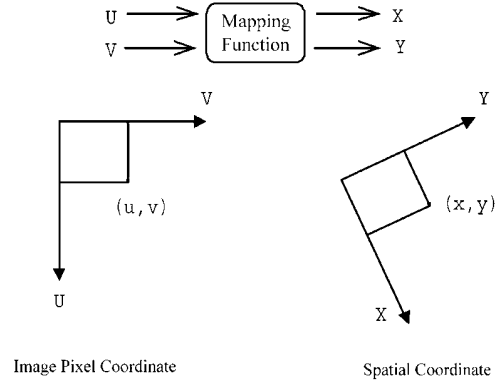

Fig. 10. The corresponding relationship between spatial and pixel coordinates.

can be found if the distances between a few spatial points, such as the four corner points of the mask, are known and the corresponding CCD image pixels are identified. Using a visual technique for measurement, every pixel on the image plane can be mapped precisely to a specific spatial coordinate. With the pixel division method, the coordinate distance between two images can then be accurately determined.

A mask, as shown in Fig. 11, with $1 \mu \mathrm{m}$ dimensional accuracy, was used to validate the proposed method. The four corner points were used as the spatial testing points. According to the predetermined spatial coordinates and measured image coordinates, regression analysis was used to derive secondorder mapping functions that will be used as the calibration scale for the grinding wheel wear assessment. The mapping functions are shown as follows:

$$
\begin{aligned}
& X=a_{1} u^{2}+b_{1} u v+c_{1} v^{2}+d_{1} u+e_{1} v+f_{1} \\
& Y=a_{2} u^{2}+b_{2} u v+c_{2} v^{2}+d_{2} u+e_{2} v+f_{2}
\end{aligned}
$$

where $(X, Y)$ is the spatial coordinate (unit: $\mu \mathrm{m}) ;(u, v)$ is the corresponding image coordinate (unit: pixel).

The derived coefficients after calibration are as follows:

$$
\begin{array}{ll}
a_{1}=0.00000 & a_{2}=0.00000 \\
b_{1}=0.00000 & b_{2}=0.00000 \\
c_{1}=0.00001 & c_{2}=0.00001 \\
d_{1}=3.75139 & d_{2}=0.03368 \\
e_{1}=-0.03506 & e_{2}=3.66189 \\
f_{1}=-313.57671 & f_{2}=-158.32445
\end{array}
$$

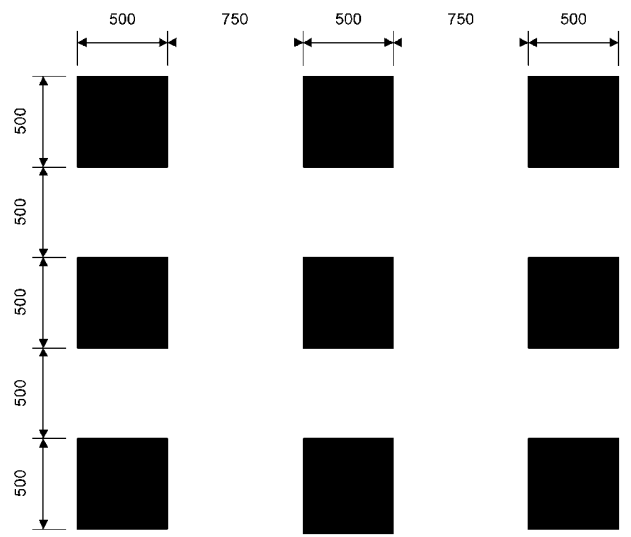

Fig. 11. Calibration mask dimensions.

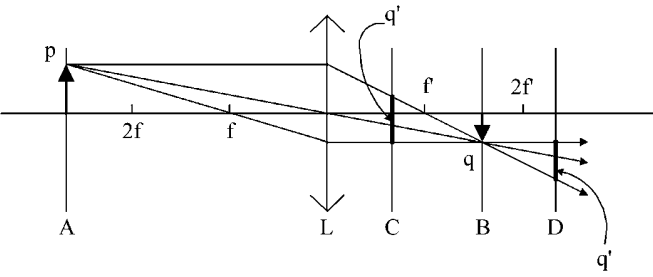

Fig. 12. The optical principle of focus and out-of-focus. A, object plane; B, focus plane; C, D, out-of-focus plane; L, lens; P, grinding wheel edge; $\mathrm{q}, \mathrm{q}^{\prime}$, grinding wheel edge image.

\section{Autofocusing}

Without proper focus, the captured image will become blurred and the edge contrast will diminish, thus the system accuracy will be degraded. An autofocus system was developed to ensure efficient image capture.

When the intensity of the incoming light varies, the CCD will generate different grey level signals. For grinding wheel edge detection, when the lens gradually loses its focus, the sharp black and white contrast at the wheel edge will become grey. Losing the focus will also create a larger grey area and the edge detection becomes more difficult.

According to optics principles, the focus and out-of-focus can be illustrated by Fig. 12. The upward arrow at plane $A$ is the measured object (grinding wheel) and point $p$ is the wheel edge. Through lens reflection and projection on the ideal focus plane, a clear downward arrow image should form at plane $B$. Point $q$ is the image of the wheel edge. When the lens deviates from its focus plane and loses its focus, for example at plane $C$ or $D$, the wheel edge image becomes a plane $\left(q^{\prime}\right)$ instead of a point. Since the energy level decreases, the image appears a blurred grey colour instead of a sharp black or white colour. As shown in Fig. 13, the grey level changes sharply at the
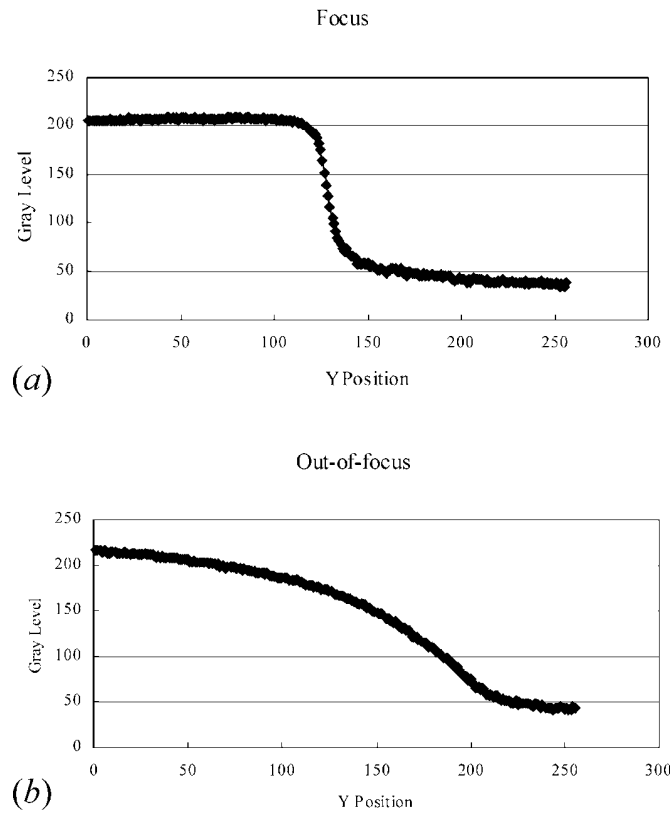

Fig. 13. Grinding wheel edge grey level comparison. (a) Focus. (b) Out-of-focus. 


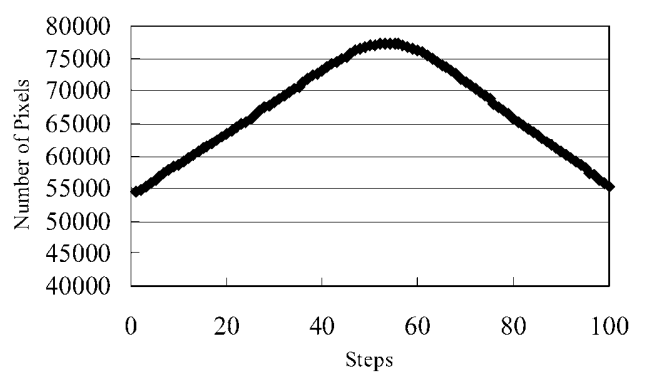

Fig. 14. Relationship between focus and number of pixels.

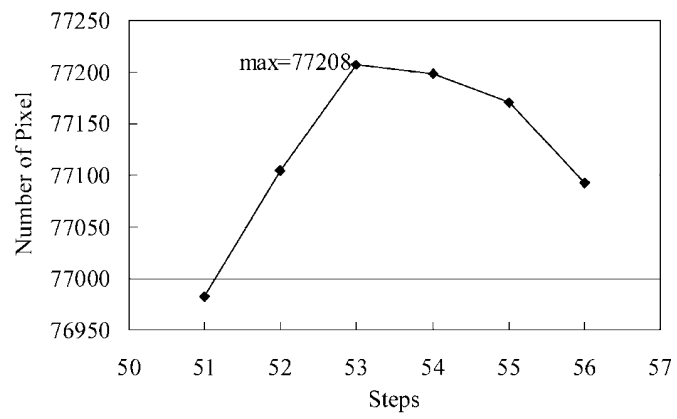

Fig. 15. Relationship between focus and number of pixels near the focus point.

wheel edge when the lens is in focus and changes gradually as an irregular curve when the lens is not in focus which leads to the formation of a grey area.

The image becomes blurred when the lens is out of focus. The image area shrinks after binarisation. When the lens is focused, the black and white image is sharp, and the number of white pixels is the largest. According to these characteristics, after binarisation, the number of white pixels can be used as a focusing index.

An experiment was conducted to bring the lens from outof-focus to focus and then back to out-of-focus. The pixels for the white area were counted and the number correlated with the number of motor rotation steps. Figure 14 shows that the number of pixels increases as the lens is focused and decreases when the focal point is passed.

Figure 15 further illustrates the change in the number of pixels near the focal point. The number of pixels is largest at step 53. However, the corresponding grey levels shown in Fig. 16 overlap with each other. Therefore, the focal point cannot be identified using grey level data.

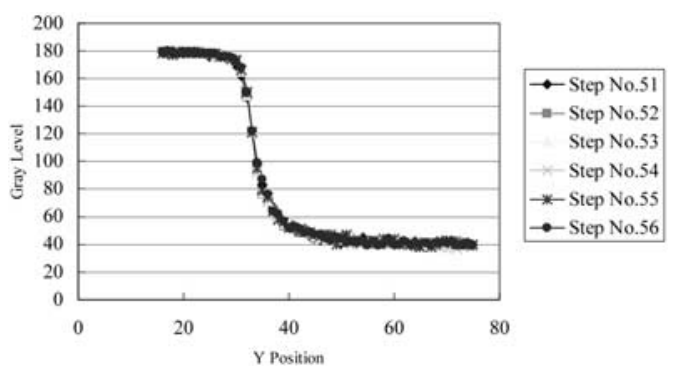

Fig. 16. Image edge grey level variation near the focus point.

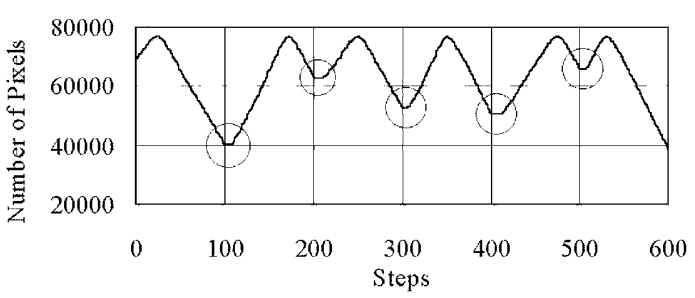

Fig. 17. Motor gear train backlash effect.

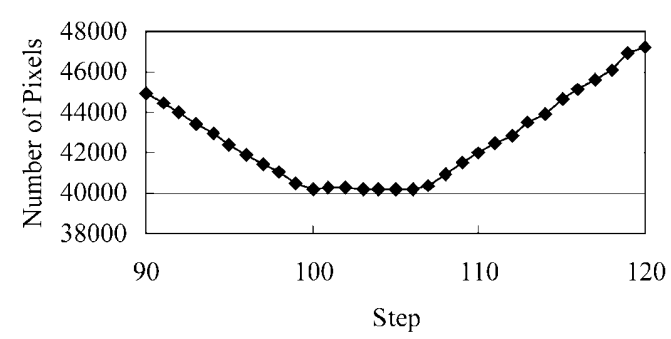

Fig. 18. Backlash for switching from clockwise to counterclockwise rotation.

In the focusing mechanism, the lens was driven by a d.c. motor through a gear train. Therefore, the backlash effect must be compensated for. Using an Intel 8255 interface card, the motor was commanded to rotate 100 steps clockwise and then reverse the rotation direction for another 100 steps. The same routine was repeated several times. By using the white image area from binarisation calibration as the index, the number of pixels will not change at the point where the rotation direction has just changed and thus the backlash effect can be quantified, as shown in Figs 17-19. The backlash error for rotation from clockwise to counterclockwise and from counterclockwise to clockwise is 6 and 10 steps, respectively.

The flowchart for autofocusing is shown in Fig. 20. To validate the autofocusing algorithm, two types of experiment were carried out. In the first type, the focus was arbitrarily and repeatedly changed and then the autofocusing routine was executed and the number of white pixels was counted. The repeatability of autofocusing is around 160 pixels. In the second type of experiment, the focus was unchanged and the white pixels of the repeat sampling were counted. The variation of pixels counted is defined as white noise and the level is around 100 pixels. Thus, comparing with the total detected pixel number of about 74460 , we could conclude that the derived autofocusing algorithm has good repeatability.

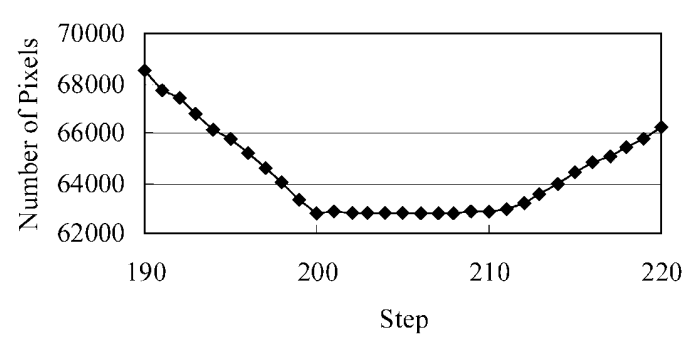

Fig. 19. Backlash for switching from counterclockwise to clockwise rotation. 


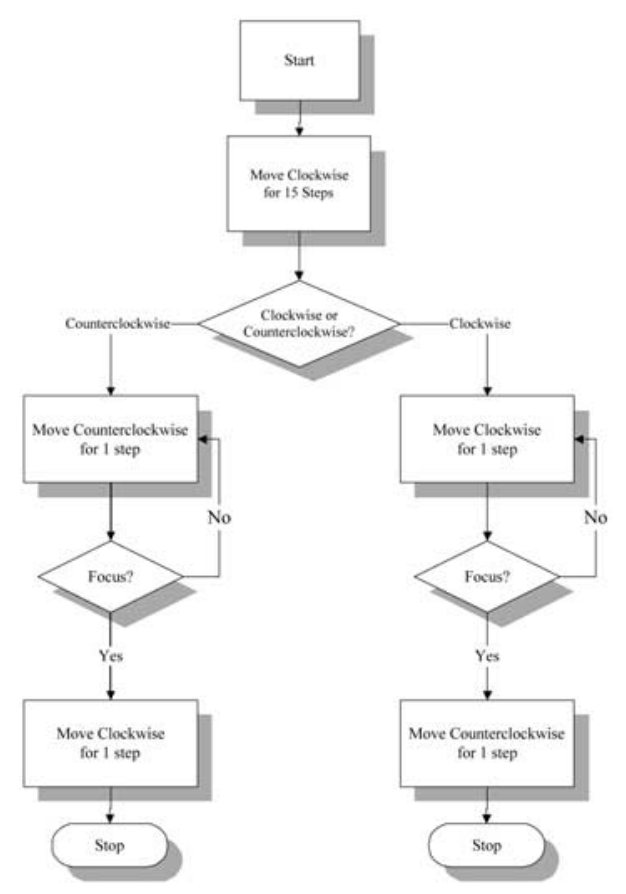

Fig. 20. Autofocusing flowchart.

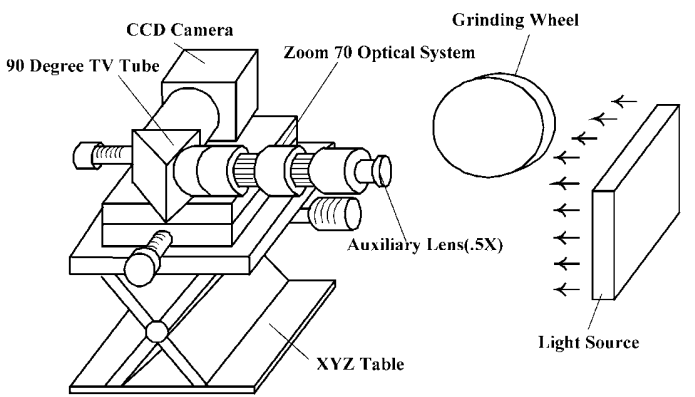

Fig. 21. Schematic diagram of the developed image capture system.

\section{Hardware}

A CCD camera was used to capture the measured object image, as shown schematically in Fig. 21. The image quality increases as the resolution increases, though the cost and the required memory capacity also increases. Since only the grey level variation is considered for image analysis, a black and white camera was selected and the specifications are given in Table 1.

The image processing speed is a concern for image card selection. The image card also needs to be activated in a

Table 1. CCD specifications.

\begin{tabular}{ll}
\hline Manufacturing company & PUNIX America Inc. \\
\hline Type & TM-7CN \\
Imager & $1 / 2 "$ \\
Pixel & $768(\mathrm{H}) \times 494(\mathrm{~V})$ \\
Shutter speed & $1 / 60 \sim / 1000 \mathrm{~s}$ \\
\hline
\end{tabular}

Table 2. Image card specifications.

\begin{tabular}{|c|c|}
\hline Manufacturing company & Imaging Technology Inc. \\
\hline Type & PC composite \\
\hline Video input & RS170/330 \\
\hline Memory & 2MB standard \\
\hline Video digitiser & $20 \mathrm{MHz}$ for $512 \times 512$ \\
\hline Capture/display rate & 30 frames $\mathrm{s}^{-1}$ \\
\hline
\end{tabular}

Windows environment and support Windows-based procedures for the ease of programming. The specifications of the image card used in this study are given in Table 2.

The characteristics of the lens used in this study are given in Table 3. The image processing resolution is defined by the ratio of object size, $h$, in lens field of view and image size, $H$, through the CCD camera lens reflection as well as the divided spatial recognition ratio of the image card. If the spatial recognition ratio can be minimised via a computation algorithm, the lens resolution can also becomes finer.

The lens field of view is $2.4 \mathrm{~mm} \times 1.8 \mathrm{~mm}$ and the spatial resolution of the image card is $640 \times 480$. Thus, the spatial resolution of the whole image capturing system is as follows:

$$
\begin{aligned}
& d X=2.4 \times 1000 / 640=3.75 \mu \mathrm{m} \\
& d Y=1.8 \times 1000 / 480=3.75 \mu \mathrm{m}
\end{aligned}
$$

In other words, each pixel corresponds to a spatial length of $3.7 \mu \mathrm{m}$. However, because of the variation in the distance between the lens and measured object, the calibration scale between pixel and actual dimension must be recalibrated.

The image capture and analysis software was developed in a Visual Basic 5.0 environment. The grinding wheel wear on-line measurement procedures involving autofocusing, lens calibration, calibration parameters calculation, and wear measurement are as shown in Figs 22 and 23.

\section{Image Measurement}

Three image measurement experiments were carried out to validate the efficiency of the proposed system. The first experiment was to measure a surface grinding wheel. The grinding wheel was installed on the spindle of a CNC milling machine, as shown in Fig. 24. The $z$ motion was used to mimic the progression of wheel wear. An HP 5529 laser interferometer was used to measure the actual displacement in the $z$-axis. The difference between the laser measurement and estimated displacement is within $\pm 0.4 \mu \mathrm{m}$, as shown in Fig. 25 .

Table 3. Lens specifications.

\begin{tabular}{lll}
\hline & Lowest & Highest \\
\hline Numerical aperture & 0.012 & 0.040 \\
Magnification & $0.38 \times$ & $2.63 \times$ \\
Field of view & $12.8 \mathrm{~mm} \times 17.1 \mathrm{~mm}$ & $1.8 \mathrm{~mm} \times 2.4 \mathrm{~mm}$ \\
Depth of field & 4.0 & 0.4 \\
Working distance & $158 \mathrm{~mm} \sim 178 \mathrm{~mm}$ & $158 \mathrm{~mm} \sim 178 \mathrm{~mm}$ \\
\hline
\end{tabular}




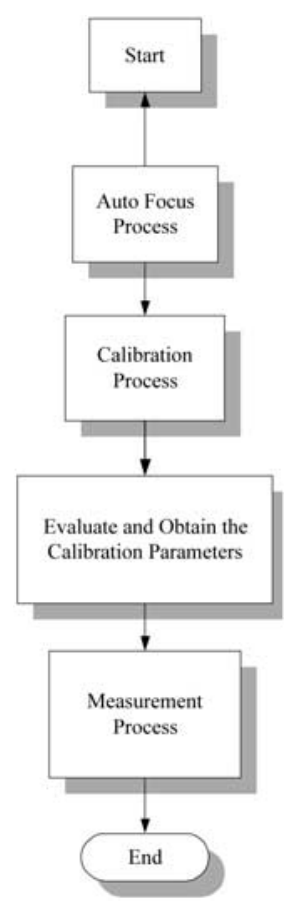

Fig. 22. On-line measurement flowchart.

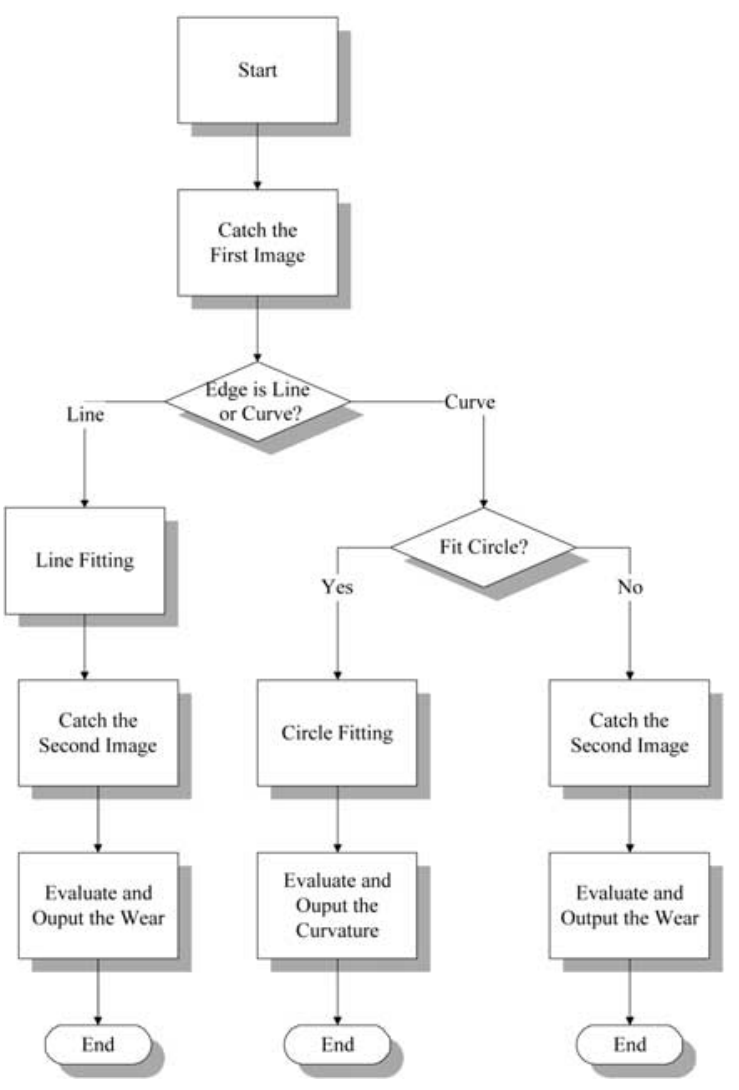

Fig. 23. Grinding wheel wear measuring flowchart.

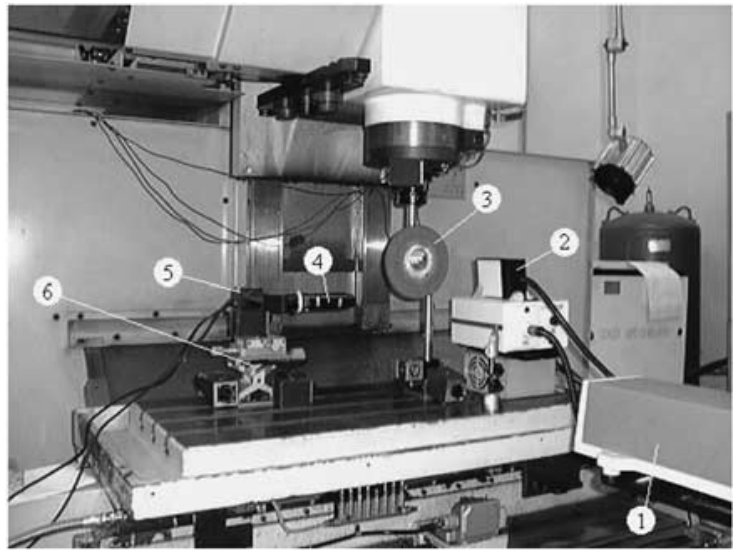

Fig. 24. Wheel image measurement experimental set-up. 1. HP5529 laser interferometer. 2. Light source. 3. Grinding wheel. 4. Zoom 70 optical system. 5. CCD camera. 6. XYZ table.

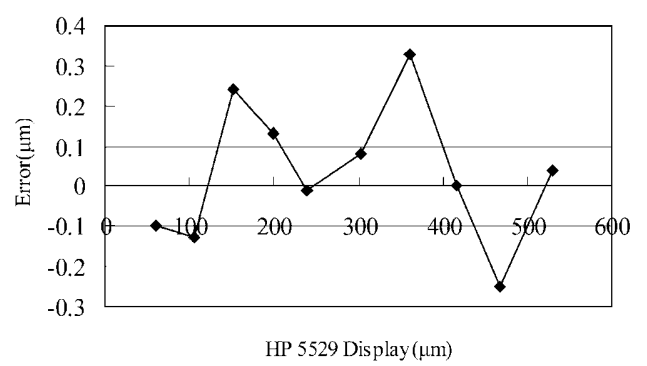

Fig. 25. Surface grinding wheel wear measurement error residual.

The second experiment was to measure a touch trigger probe head to mimic the measurement of the curved surface of a form grinding wheel. The probe head diameter has been calibrated with an accuracy of $\pm 1 \mu \mathrm{m}$. The measured diameter has a mean of $4001.50 \mu \mathrm{m}$ and a standard deviation of $2.18 \mu \mathrm{m}$. The difference between the calibrated and estimated diameters is $\pm 5 \mu \mathrm{m}$, as shown in Table 4 .

The third experiment was to measure a form grinding wheel. The grinding wheel was also installed on the spindle of a

Table 4. Residual error for measuring probe head.

\begin{tabular}{lc}
\hline Calculated value $(\mu \mathrm{m})$ & Residual $(\mu \mathrm{m})$ \\
\hline 3999.67 & -0.33 \\
4003.15 & 3.15 \\
4002.27 & 2.27 \\
4002.18 & 2.18 \\
4002.67 & 2.67 \\
3998.46 & -1.54 \\
3995.76 & -4.24 \\
4003.30 & 3.30 \\
4002.07 & 2.07 \\
4003.70 & 3.70 \\
4002.98 & 2.98 \\
4003.28 & 3.28 \\
4000.01 & 0.01 \\
4001.77 & 1.77 \\
4001.25 & 1.25 \\
\hline
\end{tabular}




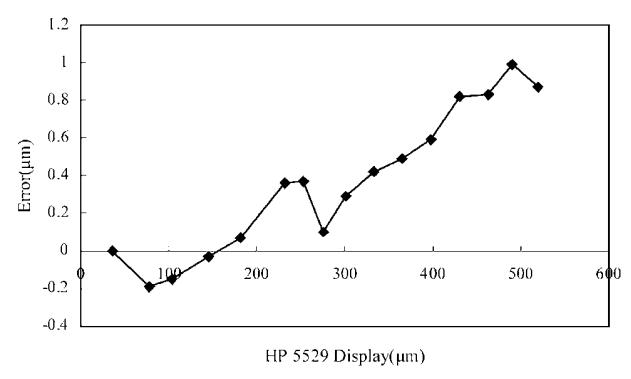

Fig. 26. Form grinding wheel wear measurement error residual.

CNC milling machine. The $z$ motion was used to mimic the progression of wheel wear. An HP 5529 laser interferometer was used to measure the actual displacement in the $z$-axis. The difference between the laser measurement and the estimated displacement is $\pm 1.2 \mu \mathrm{m}$, as shown in Fig. 26 .

\section{Conclusions}

This study used a CCD camera as a measuring device to capture the image of a grinding wheel. The image was processed using a binarisation technique and analysed using a derived edge detection algorithm to determine the spatial coordinates of the grinding wheel edge curvature. Comparing the images of the initial and worn grinding wheel, the actual wear can be assessed accurately with the desired accuracy of around $1 \mu \mathrm{m}$. The developed autofocusing system can minimise the potential error from human interaction and thus improve the effectiveness and robustness of developed system.

In summary, the following results have been achieved:

1. A binarisation technique for edge detection has been developed, leading to a significant reduction in the computational cost.

2. A pixel numbering index has been developed for a quick and repeatable autofocusing technique.

3. A Windows based user friendly measurement system, has been developed.

4. An automatic wear measurement system for form grinding wheel inspection has been developed.

According to the experimental results, we conclude that the system is very stable but the accuracy could be affected by calibration parameter derivation. Therefore, we make the following recommendations:

1. When using a standard mask for calibration, the mask should be set perpendicular to the optical axis of the CCD camera. When measuring, the cross-section of the measured object and the mask plane should overlap, to ensure the accuracy of the derived calibration parameters. For on-line measurement, the grinding wheel will be installed on the spindle of the grinding machine. Therefore, the CCD camera, calibration mask, and light source should be properly aligned to the machine coordinate system. Currently, the calibration procedure uses only 16 points on the mask for referencing. The calibration accuracy could be improved if more referencing points were used.

2. Theoretically, only a parallel light source should be used. The light source used in this system could only provide an approximated parallel light and the light source has to be very close to the grinding wheel to provide sufficient light intensity. If the light intensity is insufficient, the edge image may not be sharp enough which may lead to a measurement error.

3. The accuracy of the developed non-contact on-line grinding wheel wear image measurement system could be affected by some adverse conditions, such as machine vibration and dust in the air, in a factory environment.

\section{References}

1. Anon, Grinding Wheels and Grind Wheel Dressing, NTIS-National Technical Information Service, Springfield, Virginia, 1994.

2. J. S. Taylor, M. A. Discotty and D. A. Dornfeld, Investigation of Acoustic Emission for Use as a Wheel-to-Workpiece Proximity Sensor in Fixed-Abrasive Grinding, Lawrence Livermore National Laboratory, CA, UCRL-JC-121689, 1995.

3. I. Inasaki, "Monitoring and optimization of internal grinding process", Annals CIRP, 41(1), pp. 359-362, 1991.

4. J. F. Comes de Olicerira and D. A. Dornfeld, "Dimensional characterization of grinding wheel surface through acoustic emission”, Annals CIRP, 43(1), pp. 291-294, 1994.

5. H. Suzuki, "Development of 5 axis controlled ultra-precision grinding machine equipped with the system measuring and compensating profile error on the machine", Proceedings of Symposium for Key Technology in Precision Machine, pp. 291-294, 1995.

6. H. K. Tonshoff, "Fast sensor system for the diagnosis of grinding wheel and workpiece", SME Technical Paper, pp. 1-15, 1995.

7. R. Y. Tsai, "A versatile camera calibration technique for highaccuracy 3D machine vision metrology using off-the shelf TV cameras and lenses", IEEE Transactions on Journal of Robotics and Automation, 3(4), pp. 323-344, 1987.

8. C.-C. Wang, "A low-cost calibration method for automated optical measurement using a video camera", Machine Vision Application, pp. 259-266, 1994.

9. R. Ohlander, K. Price and D. R. Reddy, "Picture segmentation using a recursive region splitting method", Computer and Image Processing, 8, pp. 313-333, 1978.

10. B. Jahne, Digital Image Processing, Springer-Verlag, 1991.

11. Y. Shirai, Three-Dimensional Computer Vision, Springer-Verlag, 1987.

12. W.-C. Dai, "A study of three dimension machine vision scanning measurement technique", 1996, PhD Dissertation, Department of Mechanical Engineering, Chung-Yuan Christian University, Taiwan. 